\title{
THE SYSTEM OF HABITS AND THE SYSTEM OF IDEAS
}

\author{
BY ROBERT MACDOUGALL \\ New York University
}

The history of the individual mind presents, from the outset, a field of complex phenomena; it has many phases, in each of which developmental changes are occurring; with the surrounding world it has manifold points of contact; and the quantitative contents and forms of organizations by which it is characterized reflect the variety and complexity of the world in which it exists.

The student's direct approach to this complex life is beset by many difficulties. To picture it in its fulness is impossible; to combine the essential features of each phase of change in such a way as to give a view of the mind's constitution at any point in its history, is a task of scarcely less difficulty. In the face of these obstacles the psychologist has usually fallen back upon one of two procedures,-either he has considered the general form of the changes under way, as when the rate at which variation occurs is studied, and the accelerations or retardations in development are indicated; or he has selected some individual phase of change for study, as when the establishment of motor coördination is traced or the development of speech followed. This work is indispensable to a full description of mental development, and each such study finds its place in the final system of genetic psychology.

But in all genetic study, -in the description of the mind's development equally with that of organic genesis-there is more to be done than to determine the general formal changes which the subject matter presents, and to trace the development of its various constitutive functions. These studies, indeed, presuppose another form of analysis as their logical antecedent: for in every organic system which has historical and not merely logical reality, a unity of constitution is to 
be predicted. It is this assumption which underlies all elementary analysis, upon the systematic completeness of which rests the success of explanatory science generally. The assumption of a single constitutive unit and a common type of change characterizes the procedure of historical sciences at large and constitutes the ground-work for all subsequent treatment of a specialized character.

In the study of mental development, as in all other genetic science, this preliminary analysis must be undertaken, and the complex of mental changes treated in terms of the units which it yields. The student of genetic psychology thus sets out from the assumption that the qualitatively various modifications which the life of the individual mind presents are the complex products of a change the units of which are fundamentally similar throughout the whole series of phases. His first task, therefore, is to formulate these constitutive factors and to explain the system of changes in their terms.

The general character of mental development may be described as adaptation. At all stages and in every phase of its activity the change from the earlier to the later form is a reconstruction which tends to establish more harmonious relations between the individual and his environment, to restore a lost equilibrium between the forces which act upon the mind and its own responses. Adaptive reconstruction constitutes the general form of change, whether the origin of determination be conceived as lying in the environment and producing a simple adaptive modification, or the element of initiative be considered, in the utilization of materials for ideal ends. Such adaptation is incessantly renewed so long as the individual continues to live. That at one stage it leads to more systematic complexity in the reaction to stimulation, and at another stage results in a narrowing of the range of reactions, - that at one time it is constructive and progressive and at another involutionary and conservative, is a question of the special form of readjustment which occurs, and does not affect the general character of the process.

Adaptation involves two factors, a form of response already elaborated and an action tending to modify the adjust- 
ment in conformity with a variation in the system of stimuli. The former represents the level of adaptation already attained by the organism, the latter represents the increment of advance in which the fact of development consists. The first of these two factors we call Habit, the second Accommodation. Habit constitutes the response of the organism to its environment in so far as the system of stimuli possesses permanence in the course of experience; accommodation constitutes the organism's response to variations appearing within this system of stimuli.

In so far as the variation thus introduced becomes a constituent of the system of stimuli, this means simply the formation of a new habit, the development of a fixed type of response arising through a modification of those forms of reaction which previously existed, and replacing them in the economy of the mental life. In so far as concerns its outcome, accommodation thus adds nothing new, nothing which is not fully represented in the habit-system from which the process of reconstruction took its start. The movement of development is a circular one, from preëxisting habit through accommodation to later modified habit. The terms habit and accommodation, therefore, do not serve to define the opposition which is here in question, since they have application primarily to the objective reference of the mind's attitudes, and the relations which it bears to the system of outer reality.

From the psychologist's standpoint it is the immediate conscious reaction provoked by such variations in the system of stimuli as do not arouse an already established a'daptive response which must be opposed to habit and called its correlate in the system of factors constituting accommodation. This immediate reaction we call attention to the stimulus. Instead of provoking an habitual response it arouses an awareness of its own quality and of the relations in which it stands. The nature of the stimulus is thrown up in consciousness, as upon a screen, and while, in such cases, the immediate teleological response is characteristically lacking, it is through the development in a systematic consciousness of the associative complex in which the stimulus stands that the individual is led 
to that later reconstructed type of reaction which constituted the fact of accommodation. To habit is thus to be opposed attention, discriminative and selective, and the systematic consciousness to which it leads. Of this selective consciousness accommodation is a function, and from it derives the whole succession of modifications which constitutes the course of mental development.

The two constituents of mental development may thus be described as the system of habits and the system of ideas. It is the progressive organization of a system of habits and the continuous elaboration of a system of ideas. Each of these factors has a specific function and a continuous history in mental development. The system of habits represents the organism's response to the permanent aspect of the environment; the system of ideas represents its response to the variational aspect. Wherever the system of stimulations presents recurrence, the organism responds with a habit; wherever that system presents novelty the organism responds with an idea.

At every stage of its history these two factors are involved in the mind's activity. There is no consciousness which is not determined by preëxisting habit, nor is there any habit which is not subject to modification through the consciousness which accompanies it. On the other hand, the relation of these two factors determines the character of any experience; and their concomitant variations mark the range of activities by which the mental field is characterized. In those provinces within which the relations between the organism and its environment are highly defined and maintained with relative continuity, the points at which consciousness is aroused are few and unimportant. The element of habit constitutes the dominant feature of the situation, and awareness of the qualitative character of the stimulus falls to its lowest ebb. Where, on the contrary, such relations are ill-defined and subject to frequent and profound alterations, a fixed type of response does not supervene, but attentive consciousness attains its greatest acuteness.

These two constituent factors of development appear in 
every field of mental activity, and throughout its range maintain their fundamental significance unmodified. The function of genetic psychology is to define the place and contribution of each factor in any given phase of mental activity, and to trace the evolution of the organic system to which the two elements lead in their respective spheres.

Thus in the description of the system of perceptual and of conceptual objects alike, both of these factors are found indispensable. The response to each object of perception involves habit, since reaction to such stimuli depends upon the recurrence of typical sensation complexes in the individual's experience, the momentum of habituation in the reaction varying with the fixity of these complexes and the frequency of their recurrence. In so far as this element of routine prevails, the adaptive reaction provoked by the stimulus is automatic and unaccompanied by consciousness. In so far as the field within which the stimulus lies is subject to fluctuations, and the relations between it and the organism are incessantly disturbed, the reception of the stimulus and the reaction to which it leads will be pervaded by consciousness.

It is sufficiently obvious that the system of objects which constitute the field of perception will not become the source of a series of purely automatic adaptations. The calling forth of a wholly unconscious and habitual response will be approached only in cases where a great simplicity in the stimulus obtains, and where a high degree of uniformity in the conditions of its occurrence is possible,-as in certain mechanized signals of rhythmical recurrence with which an unvarying response is connected. If this type of reaction developed in connection with the general system of stimuli which the sensible world presents, the individual would constantly be involved in disastrous situations through variations in the concomitants of the particular stimulus which conditioned his response. There is perhaps no conceivable situation for which a purely automatic reaction constitutes a sufficient adaptation, since from none is the possibility of significant variation at any moment absent. The reactions 
connected with even the most familiar objects are subject to incessant subtle modifications; and the possibility of these modifications must at every instant be taken into account in the formulation of the response.

This potential variability in the stimulus conditions what we call attention to its character at each recurrence. Perception, in a word, represents the margin of variability in the field of the stimulus. The response which the individual makes to sensible objects thus involves both attention and adaptive movement; and its elaboration gives rise on the one hand to a system of habits and on the other to a system of ideas.

In the field of conceptual objects the same phases appear. This is perhaps best illustrated by the form of the individual's reaction upon the familiar terms of speech. The response to significant speech involves the element of habit in so far as each word provokes an unreflective response in terms of the adaptation, or meaning, which it represents. The warning may thus be acted upon without distinct awareness of the words which have prompted the action. It is only when meaning is doubtful, when words are ambiguous or construction obscure, that attention is directed to the form of expression, and the margin of variability in the stimulus is developed in a critical review of the possible applications of the term.

But reflection shows that grammatical construction, at least, is always variable; and that habituation can reach a high degree of development only in connection with common words and their uses, or at most with certain simple and unvarying constructions which are in frequent use. Such are the current verbal coin of polite intercourse, the forms of which are stereotyped and become devoid of specific significance, and, on the part of user and hearer alike, lapse into practically complete automatism. In the case of all ordinary human intercourse, significant speech is essentially mobile and variable. Every element has a meaning of its own, which constitutes, in a sense, the core of the whole system of meanings connected with it; but as an element in a sentence it has a meaning which is defined only 
by the context in which it is found, and cannot in any way be determined by its signification in isolation. Even when used without context it is rarely, if ever, that a term is univocal, and that it may be responded to by a single unvarying reaction.

In any case, then, response to the forms of human speech involves incessant attention which must continue throughout its course. The development of the margin of variability ceases only when the expression is brought to a close, since the meaning is constructively developed, as the terms are apprehended in succession, by a progressive modification of the whole preceding context. Every syntactic form is thus typically unique, with the exception, noted above, of such expressions as have become stereotyped; so that it is logically inconceivable that an automatic response should ever be established in connection with it. It is this character which makes human speech so much more stimulating than the perception of the sensible world. The latter approximates the values of speech as an ideal stimulus only in those extreme cases which may be termed crises, namely such as call for immediate and difficult coördination, danger, personal encounters, the control of active and variable forces, and the like. In the apprehension of human speech the margin of variability being greater and of more continuous importance, the system of ideas attains a higher functional development than that which it presents in connection with the perception of the external world. Speech provokes an incessant, lively and variable play of ideas which is the necessary consequence of the fact that from its very nature speech cannot indicate its object intuitively, but must make it known through a system of discursive symbols.

A similar quality appears in the relations which may exist between any mental image, or any concept, and the context with which it is connected in individual thought. The connections linking a series of ideas which successively occupy the mind may be so simple and variable that each suggests the next in an immediate and inevitable manner and the last of the series may lead to specific reaction without any 
reflective consciousness concerning the internal constitution of the ideal system by which its occurrence 'was mediated. Only the terms of the series, as the event is recalled in afterexperience, seem to have been in mind, the intermediate elements, whether one or many, having apparently dropped out of existence. It is not necessary, in such cases, to deny that mental activity of any kind has occurred, but if the occurrence of a succession of ideas be granted, it has conformed so closely to the habit type that attention to the constituent elements has fallen to its lowest ebb, and the margin of variability which conditions consciousness is practically negligible.

Such successions are possible only when the order of associations is extremely familiar, when thought is following some thoroughly trodden path. In every mind such idiosyncratic forms are developed, and all permanent social life fosters the establishment of common ideal types which tend inevitably to the form of habit-modes in popular usage. Such ideas are received uncritically and provoke immediately certain specific mental attitudes in relation to their object which also commonly prompt stereotyped communal expressions. Every human group owns such authoritative conceptions,--ideas and symbols made sacred by religious or political or moral sanction-which by common consent have been raised above the stage of debateableness and made sacrosanct in the thought of the multitude. These conceptions constitute the idols of the forum and of the marketplace, which drug men's minds instead of stimulating them. They silence thought by demanding an immediate emotional or practical response of an invariable type. Reflection has been interdicted in connection with concepts which have reached this status and criticism is anathematized. They thus become, as in the case of much of the technical terminology of sectarian religions, merely cant phrases, employed not to stimulate the development of meaning but solely to provoke a typical habitual response, namely the specific mood or act which tradition has connected with the verbal formulation.

In this system of ideal conceptions, and in the understand- 
ing of human speech as well, the function of habit is the same as that which it fulfills in the field of perception and motor reaction. It represents the system of fixed and permanent relations which finds place within each province of mental activity. These highly defined and recurrent aspects of the system of conceptions constitute the basis of rationality in our thinking. Repetition is essential to the organization of the field of consciousness as variation is to its preservation. In proportion as the relations among the mind's objects are ascertained and made familiar through repetition, they cease to occupy the attention. In the mind's procedure they still have a place, but they are neither reflectively formulated on the occasions of their employment, nor do they detain the mind at their occurrence and thus become the center of a new associative system. Conception and equation, formula and deduction follow one another in a typical habit series when they have thus reached full definition and attained final familiarity for the mind which employs them.

The system of relations represented in the multiplication table affords an illustration of this process of habituation. The relations here are simple and invariable, and while the establishment of the system in mind may involve sustained effort and a high degree of selective attention, the tabulation of this series of relations and the study of them by the individual has fulfilled its object only when the mind has been so thoroughly familiarized with the whole system that the product of any two numbers arises immediately and unreflectively upon the presentation of the numbers in question. In the final stage of this development there is no multiplying process interposed between the thought of the numbers and the thought of their product, to which the thinker can point. The procedure has been so mechanized that in the case of the expert a series of such simple operations may be performed without interrupting the continuity of attention to other objects of thought.

Just as in the case of human speech it is essential that the fundamental meanings should be mastered in such a degree that their signification needs not to be established anew on the 
occasion of each recurrence, so in the general system of concepts by means of which the mind works, it is indispensible that group after group of formulations should thus be automatized if the mind is to advance in its task of reducing the phenomena of experience to logical order through the application to them of an ascending series of ideal conceptions.

In all phases of the mind's activity, whether it be motor co-ordination, or perception of the external world, or associative thought, the same phenomena are presented. At any stage in its development there is given an existing system of habit-modes which is under continuous extension and organization through a process of selection and reconstruction. The system of existing habit-modes we may call the individual mind; for the latter term is commonly employed to denote the specific characters of the individual,-those mental features which, though they undergo change, present at any time such definite forms that they constitute a synthetic type by which the individual may be recognized and described. The selective and reconstructive modification is the function of individual consciousness, which arises at those points where equilibrium has been disturbed, and mediates the new form of reaction which is in process of formulation. The interaction of individual consciousness and individual mind constitutes individual life, which is a process of continuous re-adaptation in a cyclical type of change, wherein, through the disturbance of equilibrium between an older settled habit and the environment to which it is adapted, an activity of discriminative and selective consciousness is initiated, leading to the establishment of new habit-modes through modification and re-organization of the old in an endless succession.

In this whole process the terminus to which the reconstructive activity tends is unconscious automatism. In its final phase it is an adaptive reaction, in the form of some specific movement or bodily attitude: it is never a system of ideas. The system of ideas, whether feelings of bodily condition, perception of external objects, mental images, or conceptual forms, is that phase of the mind's existence which mediates the formation of habits. The system of ideas cannot 
itself exhibit habit; just in so far as habit supervenes the ideal content of experience declines. It is thus not proper to say that the system of ideas as well as the system of movements is subject to habituation. On the contrary, the system of ideas exists in so far, and only in so far, as the conditions of habit-forming fail within any given field of experience. The system of ideas represents the margin of variability in the world of experience at large. It stands for those elements and aspects which are not yet coördinated with, and capable of immediately provoking, appropriate adaptive reactions. The system of ideas thus reflects the outer circle of environmental conditions, the world of problematic relations and unsolved adaptations. It is the field of doubt and hesitation, of stress and struggle, the field within which, though the object of the will has already been formulated, the means to its elaboration have not yet found definition, and the mind is occupied with an incessant vivid representation of the alternative but still dubious courses which the situation suggests.

The vividness of consciousness is intensified wherever the form of habit suffers disturbance, as well as where it is primitively lacking. In the child's pristine experience every detail of those events which in later years we call hum-drum is eagerly welcomed; and if the sense of living experience decline with age, it is because the extension of habit in life has not been accompanied uniformly by a correlative development of interest in novel forms of stimulus. It is conceivable that the system of organized habits should approach so near to the confines of experience as to leave only a negligible element of ideal activity, confining the individual in a narrow round of routine, in which his dull and brutish soul is illuminated by only an inconstant gleam of speculation. It is conceivable also, that in a fortunately born nature the order of experience should remain so full of possible alternatives that not even the most trivial occurrence has completely lost suggestiveness, so that an aura of serious or whimsical speculation surrounds it in the fulgurating mind. Such individuals never grow old, we say; and their youthfulness lies just in this freshness of vision which enables them to avoid 
the shackles of intellectual habit, and to maintain a child's originality in their treatment of the increasingly complex phenomena which the course of experience presents.

Duller minds are touched by this deepening and quickening of existence wherever their habitual routine is disturbed, as when a hitherto unconsidered doubt is cast upon some settled belief, or a familiar fact is presented in a novel but consistent way. Whether the sharpened sense of experience be found exhilarating or poignant will depend upon the specific direction of departure from the fundamental habits of life. But, in both cases alike, the experience is characterized by the sudden flooding of a field of organized and habitual attitudes with an acute and vibrant consciousness.

The normal relation of these two systems of factors in mental development may be stated briefly. The system of habits gives to ideal activity its point of origin and its direction: the system of ideas gives to habit a telic value, and maintains its commensurability with an enlarging environment. Without habit experience would be an irrational chaos; without ideas it would have no existence,-for experience is not posited of an organism in virtue of adaptation in general, but only in so far as adaptation constitutes an ideal process determined by a specific aim and a sense of value. Normal development in the individual mind, therefore, is that process of change which leads towards a more complex synthesis of habit-modes and a widened ideal horizon; and the highest type of self is that in which a life of the most intense intellectual activity finds at once its basis and its object in the fullest organization of experience in terms of significant reactions of the will. 